$11-2019$

Meeting the challenges of exsanguinating haemorrhage ; $\mathrm{A}$ balloon as a 'bridge'

Zia Ur Rehman 


\section{Meeting the challenges of exsanguinating haemorrhage; A balloon as a 'bridge'}

Zia Ur Rehman

Madam, Major uncontrollable bleeding in trauma can be compressible (extremity trauma) or non-compressible (chest or abdominal cavity). Non-compressible bleeding mostly arises from intra-abdominal vascular injuries or injuries to the solid organs. Since direct pressure cannot be applied to control this type of haemorrhage, patients can rapidly become haemodynamically unstable. The placement of an endovascular aortic balloon can tamponade proximal to the bleeding vessel site and can stop the bleeding before irreversible shock ensues. It can act as a 'bridge' to definitive treatment. By occluding the aorta, blood pressure increases and fluid requirement decreases. This is named as Resuscitative endovascular balloon occlusion of aorta (REBOA).

Resuscitative thoracotomy and aortic cross clamping was conventionally advocated in these situations for achieving temporarily control of the bleeding but was associated with a high morbidity. ${ }^{1}$

The REBOA can be inflatable at three aortic zones. Zone 1 is in the thoracic aorta from subclavian artery down to coeliac artery while Zone 3 is between the lowest renal artery to aortic bifurcation. ${ }^{2}$ It is placed in Zone 1 for active intra-abdominal bleeding while in the Zone 3 in case of pelvic bleeding of lower extremity haemorrhage. Zone 2 contains origin of visceral and renal vessels and is not recommended for balloon tamponade for control of intrabdominal bleeding.

The femoral artery is access of choice for placement of REBOA catheter due to its relative good size, superficial position and easy approach. Left brachial and carotid

Department of Surgery, Aga Khan University Hospital, Karachi Pakistan.

Correspondence: Zia Ur Rehman e-mail: drzia7179@gmail.com arteries are the alternative approaches.

Despite its potential huge role, data is lacking to demonstrate clear benefits of ROBEA in reducing overall morality of the trauma patients.3,4 This may due to practical difficulty in conducting a large clinical trial for critically injured patients. There is also discussion going on to identify the group of patients who may benefit most from the use of this technique and in which patients it is best avoided. There are access related complications and also it is not recommended in case of thoracic aortic injury due to its potential to exacerbate it.

ROBEA is a novel concept which have potential role in the management of these massively bleeding patients.

Disclaimer: None to declare.

Conflict of Interest: None to declare.

Funding Sources: None to declare.

\section{References}

1. DuBose JJ, Scalea TM, Brenner M, Skiada D, Inaba K, Cannon J, et al; AAST AORTA Study Group. The AAST prospective Aortic Occlusion for Resuscitation in Trauma and Acute Care Surgery (AORTA) registry: Data on contemporary utilization and outcomes of aortic occlusion and resuscitative balloon occlusion of the aorta (REBOA). J Trauma Acute Care Surg 2016; 81: 409-19.

2. Stannard A, Eliason JL, Rasmussen TE. Resuscitative endovascular balloon occlusion of the aorta (reboa) as an adjunct for hemorrhagic shock. J Trauma 2011; 71: 1869-72.

3. Norii T, Crandall C, Terasaka Y. Survival of severe blunt trauma patients treated with resuscitative endovascular balloon occlusion of the aorta compared with propensity score-adjusted untreated patients. J Trauma Acute Care Surg 2015; 78: 721-8.

4. Moore LJ, Brenner M, Kozar RA, Pasley J, Wade CE, Baraniuk MS, et al. Implementation of resuscitative endovascular balloon occlusion of the aorta as an alternative to resuscitative thoracotomy for noncompressible truncal hemorrhage. J Trauma Acute Care Surg 2015; 79: 523-30.

doi: 10.5455/JPMA.26435. 